\title{
SUR LES LARVES D'ANOPHÈLES RÉCOLTÉES EN CORSE AU COURS DE L'ETÉ 1959
}

\author{
Par M. SICART et J. RUFFIE
}

Au cours des vacances 1959, passées en Corse, nous avons eu l'occasion de récolter 4.346 larves d'Anophèles, dont 1.395 étaient au stade IV. Seules ces dernières furent retenues pour la présente étude.

Les gîtes visités se situent dans la presque totalité de l'île, sauf toutefois dans la zone montagneuse que, faute de temps, nous n'avons pu visiter. Cependant, le plus grand nombre de gites prospectés se trouvent sur la côte orientale, principalement dans la région de Porto-Vecchio.

Nous indiquons succinctement ci-dessous les différents espèces et biotypes rencontrés.

ANOPHELES MARTERI.

\section{(6 larves)}

Un seul gîte près de Ponte-Leccia, à une altitude de $500 \mathrm{~m}$. Gîte classique, eau claire, dans un abreuvoir entretenu par une source minuscule. Peu de végétation. D'ailleurs, les larves de cette espèce se tiennent plutôt sur la rive et n'hésitent pas lorsqu'elles sont effrayées à remonter dans le sable de la rive au lieu de plonger. C'est ce qui explique en partie la rareté de leur capture.

\section{ANOPHELES ALGERIENSIS.}

\section{(9 larves)}

Tous les gîtes rencontrés sont des gîtes de plaine. Leurs caractéristiques sont diverses : eaux claires ou troubles, ensoleillées ou non, avec ou sans végétation. Deux fois, nous les avons trouvées cohabitant avec des larves d'A. claviger. Pas de remarques notables, la rareté de cette espèce pouvant la faire considérer comme négligeable sur le plan de l'épidémiologie.

ANOPHELES CLAVIGER.

(91 larves )

Il s'agit de larves au stade IV trouvées dans 16 gites répartis sur toute l'île. 


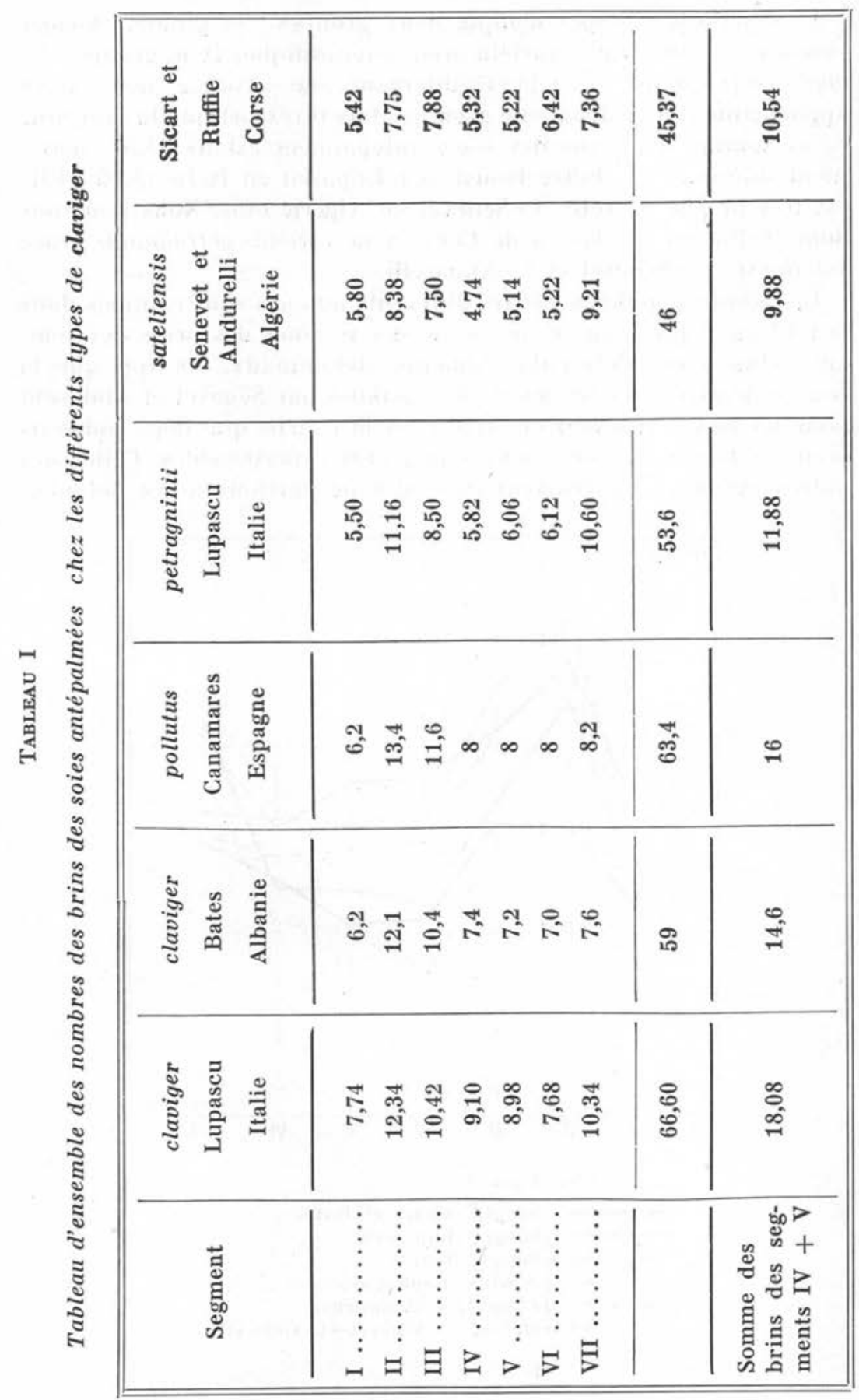


Le complexe claviger compte deux groupes: le groupe claviger claviger (= missiroli), variété atlantico-nordique, et le groupe claviger petragnanii, variété méditerranéenne. Toutes nos larves appartiennent à ce deuxième groupe. Mais il ressort que la moyenne de la somme des brins des soies antépalmées est de 45,37 , nettement inférieur au chiffre trouvé par Lupascu en Italie $(53,6)$. Elle est très proche de celle de Sénevet en Algérie (46). Nous pourrons donc rattacher les larves de Corse à la variété petragnanii, race sateliensis, G. Sénevet et L. Andarelli.

Les résultats publiés par les différents auteurs sont résumés dans le tableau 1 qui donne la moyenne des sommes des brins des soies antépalmées des différents segments abdominaux. On voit que la courbe dressée à partir des chiffres publiés par Sénevet et Andarelli pour les larves trouvées en Algérie et la courbe que nous publions pour les larves de Corse sont à peu près superposables. Celles des autres auteurs s'en écartent dans des proportions assez notables.

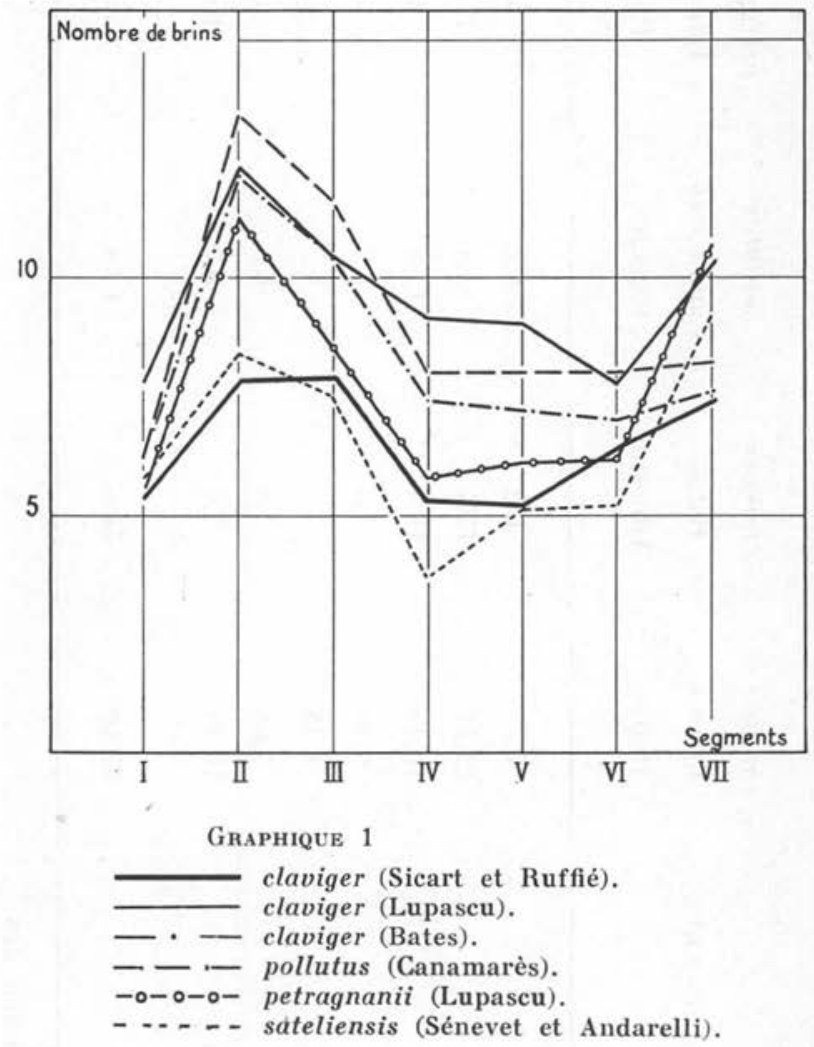


Nous indiquons ci-dessous la liste des gîtes à A. claviger que nous avons visités :

\section{Secteur}

Calvi $\ldots \ldots \ldots \ldots$

Porto-Vecchio ....

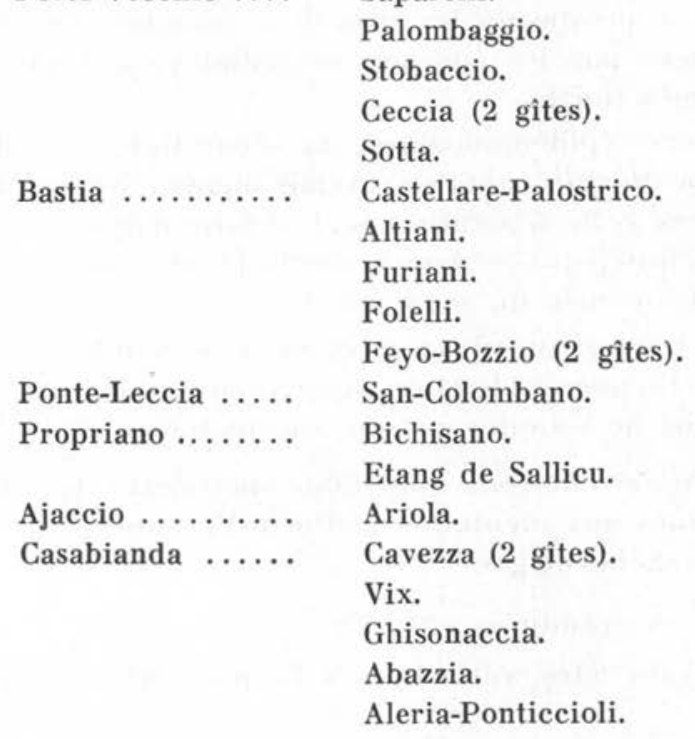

D'après les constatations faites par l'un de nous en Afrique du Nord, nous pensons que la variété petragnanii est une race biologiquement caractérisée par son anthropophilie. Nous nous trouvons ici en présence d'un facteur non négligeable pour la transmission du paludisme, puisque très répandu dans toute l'île, et nous estimons que la lutte contre le claviger doit être menée avec autant de rigueur que contre le labranchiæ.

De plus, A. claviger est un moustique exophile qui ne sera pour ainsi dire pas atteint par les méthodes de lutte anti-imago; donc, la lutte antilarvaire est seule à envisager.

Les gîtes de $A$. claviger ont des caractères très divers. En règle générale, les larves se tiennent dans les mares sans végétation ou avec une végétation horizontale peu dense. Les gîtes ne sont jamais très importants, l'eau en est froide et claire. 
Complexe MACULIPENNIS.

(1.294 larves au stade IV)

(Nous n'avons pas retenu pour notre étude les stades plus jeunes).

Ces larves étaient réparties inégalement dans une quinzaine de gìtes. Tous les gîtes sont cantonnés dans la plaine (nous n'avons pu, faute de temps, prospecter les gîtes de montagne). De plus, par suite du traitement par les équipes antipaludiques, beaucoup de gìtes se sont révélés stériles.

Au point de vue épidémiologique, la séparation des diverses espèces du groupe Maculipennia se révélait pleine d'intérêt. On sait que, chez les larves, cette séparation peut se faire d'après le nombre de brins de soies antépalmées des segments IV et V et l'aspect des folioles de la soie palmée du segment II.

Nous n'avons retenu que deux gites dont le nombre de larves récoltées permettait une étude statistique valable. Les autres étant trop pauvres, nous ne les citerons que succinctement.

$1^{\circ}$ Porto-Vecchio et environs immédiats (périmètre de $5 \mathrm{~km}$.). Il s'agit de gîtes situés aux alentours de Porto-Vecchio, dans les jardins et forêts de chênes-lièges.

Nombre de larves examinées : 234 ;

- larves pouvant être rattachées à l'espèce labranchiæ : 200. soit $85 \%$ :

- larves pouvant être rattachées à l'espèce typicus : 34 , soit $15 \%$.

Nous savons que les biotypes labranchiæ et atroparvus présentent sur le segment II une soie palmée rudimentaire de 10 à 18 folioles étroites; chez la race sicaulti, cette soie est piliforme. Ces trois types portent sur les segments IV et V des soies antépalmées d'un à quatre brins.

Si nous additionnons le nombre des brins des soies antépalmées sur les deux segments, nous arrivons au tableau 2 suivant:

D'autre part, nous avons relevé la présence de 34 larves de typicus présentant une soie palmée piliforme sur le segment II et des soies antépalmées sur les segments IV et V présentant un nombre de brins de 5 à 12 , avec une moyenne de 6,29 .

Dans nos récoltes, nous n'avons trouvé que des œufs de labranchiæ et de melanoon. Nous devons donc rattacher à l'espèce labranchiæ les 200 larves présentant une moyenne de 2,29 brins par 
TABleau 2. - L'histogramme 1 résume nos résultats

\begin{tabular}{|c|c|c|}
\hline Nombre de brins & Nombre de larves & $\%$ \\
\cline { 2 - 3 } 4 & & \\
5 & 4 & 2 \\
6 & 8 & 4 \\
7 & 5 & 2,5 \\
8 & 12 & 6 \\
9 & 49 & 24,5 \\
10 & 42 & 21 \\
11 & 33 & 16,5 \\
12 & 28 & 14 \\
13 & 15 & 7,5 \\
14 & 2 & 1 \\
& 2 & 1 \\
\hline \hline
\end{tabular}

Histogramme 1

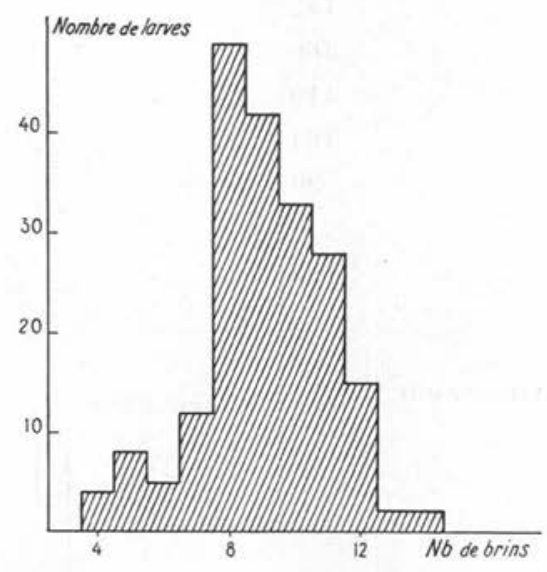

soie antépalmée, et à l'espèce maculipennis, var. melanoon, les 34 larves présentant une moyenne de 6,29 brins par soie antépalmée.

$2^{\circ}$ Le deuxième gîte étudié et le plus important se trouvait dans un jardin à $4 \mathrm{~km}$. environ de Sotta (localité légèrement au SudOuest de Porto-Vecchio). 
Ce gite, loin de toute habitation, a étẻ conservé pendant quelque temps à cause de la diversité des espèces rencontrées (11 espèces ou variétés de Culicidés) et des larves d'Anophèles nécessaires à notre expérimentation.

Le nombre des larves examinées a été de 992, dont :

- 10 larves de melanoon, soit $1 \%$;

- 982 larves de labranchiæ et sa variété sicaulti, soit $99 \%$.

Pour l'espèce labranchiæ, la répartition des brins des soies antépalmées des tergites IV et $\mathrm{V}$ était la suivante (tableau 3 ) :

TABLEAU 3. - L'histogramme 2 résume ces résultats

\begin{tabular}{||c|c|c||}
\hline \multirow{2}{*}{ Nombre de brins } & Nombre de larves & $\%$ \\
\cline { 2 - 3 } 4 & 13 & $\%$ \\
5 & 20 & 1,3 \\
6 & 51 & 2 \\
7 & 82 & 5 \\
8 & 222 & 8,3 \\
9 & 182 & 22,5 \\
10 & 160 & 17,6 \\
11 & 119 & 16,6 \\
12 & 104 & 12,1 \\
13 & 20 & 10,5 \\
14 & 7 & 2 \\
15 & 2 & 0,7 \\
& & 0,2 \\
\hline
\end{tabular}
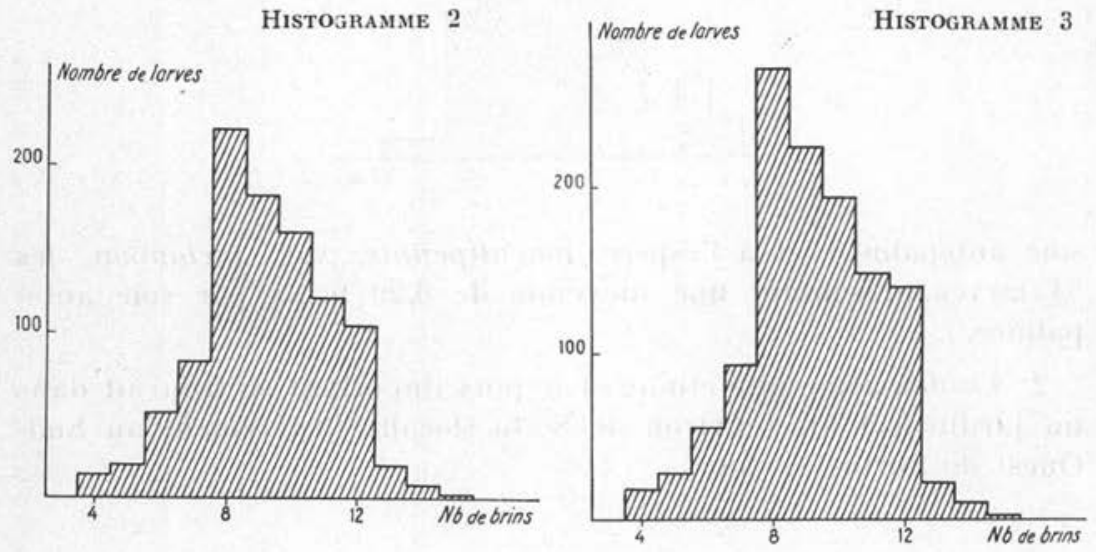
La moyenne des brins d'une soie antépalmée est de 2,289, comme dans le gìte précédent. La comparaison des deux histogrammes démontre que les lots étudiés sont tout à fait remarquables en ce qui concerne la répartition des brins des soies antépalmées. A partir de ces deux lots, on a pu construire l'histogramme 3.

Les autres larves pouvant être rattachées au typicus présentent toutes une soie palmée piliforme sur le segment II et des soies antépalmées sur les segments IV et $\mathrm{V}$ qui portent un nombre de brins de 4 à 7 avec une moyenne de 5,40 et un total minimum de 20 pour les quatre soies.

Dans ce gîte, nous avons récolté des œufs appartenant à une espèce, le labranchiæ, et sa race ovigère, sicaulti, ainsi qu'à la variété melanoon, rattachée au typicus.

La présence d'œufs de sicaulti mérite d'être signalée, car, à notre connaissance, c'est la première fois que cette race est trouvée en dehors du Maroc.

Nous avons recherché les caractères différentiels (1) entre les larves labranchiæ et sicaulti chez nos larves récoltées dans le gîte de Sotta.

Sur les 982 larves examinées, nous en avons trouvé 16 qui présentaient d'une façon indiscutable des soies antépalmées piliformes sur le segment II, ce qui donne une proportion de $1,6 \%$. Nous avons noté aussi que la soie palmée du segment II présentait des variations des folioles assez notables quant au nombre et à la largeur. Chez certaines larves, cette soie est presque normale, avec folioles à peine plus étroites que la soie du segment sous-jacent. Chez d'autres, les folioles sont en nombre réduit (9-10) et elles sont étroites.

De plus, la moyenne des soies antépalmées des segments IV et V est de 2,49 (1 à 5 ), très proche de la moyenne générale que nous avons calculée plus haut. Il semble donc que c'est uniquement le critère de la forme des folioles de la soie palmée du segment II qui permet de séparer la race sicaulti de l'espèce labranchiæ.

Dans le gìte étudié, la proportion des larves de cette race est très faible par rapport à l'ensemble de la population, et nous ne pouvons qu'attribuer au hasard la découverte de deux œufs présentant tous les caractères sicaulti.

Cela permet de penser que cette race n'est pas uniquement marocaine, mais qu'elle s'étend sur tout le pourtour Sud de la Méditerranée Occidentale. Sa rareté la fait seule passer inaperçue.

(1) Nous remercions bien vivement $\mathrm{M}$. le $\mathrm{P}^{\mathrm{r}}$ Roubaud, à qui nous avions signalé ce fait et qui a bien voulu nous donner ces caractères. 
Au point de vue épidémiologique, elle est, comme l'espèce labranchiæ, un vecteur important du paludisme et ne doit donc pas être séparée de cette dernière.

$3^{\circ}$ Les autres gîtes larvaires à Maculipennia se trouvent dans les secteurs suivants (toujours larves au stade IV seulement) :

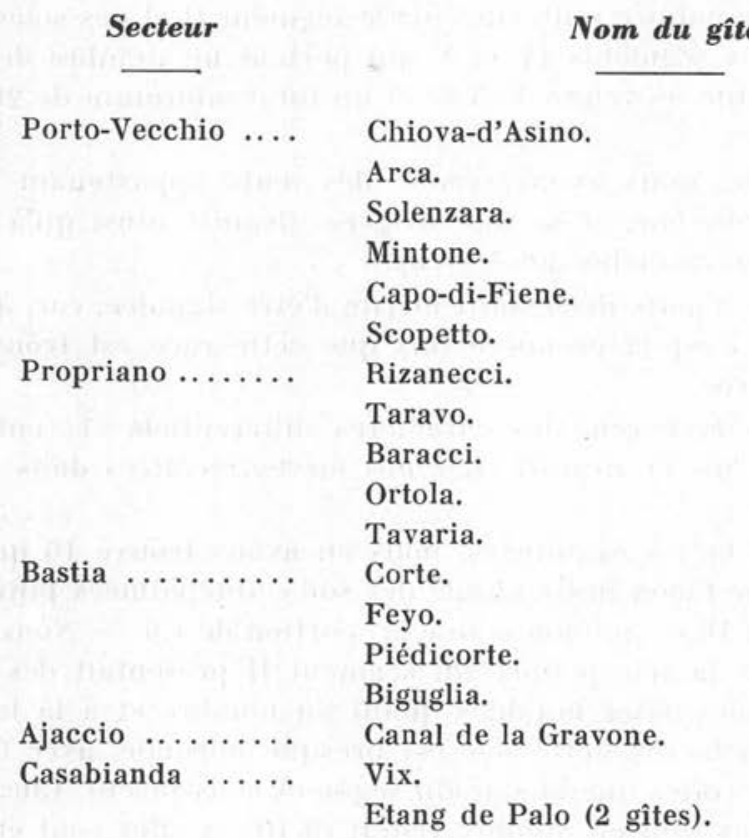

En résumé, nous avons noté la présence d'Anophèles vecteurs sur toute l'étendue de l'île.

De plus, nos examens nous ont permis de déceler la race sicaulti mêlée en faible proportion à l'espèce labranchiæ.

Cette constatation permet d'étendre l'aire d'expansion de cette forme que l'on pensait jusqu'à présent limitée au Maroc. A notre avis, on doit aussi la rencontrer dans les pays bordant le Sud de la Méditerranée Occidentale.

Quant à Anopheles claviger, la variété colonisant la Corse est la variété petragnanii forme sateliensis, variété à tendances anthropophiles, donc dangereuse.

Travail du Laboratoire de Parasitologie de la Faculté de Médecine de Toulouse ( $\mathrm{P}^{r}$. L. Boursset) 